\title{
Study of Some Sequences of Prime Numbers Defined by Iteration
}

\author{
Idir Sadani* \\ Department of Mathematics, University of Mouloud Mammeri, Tizi-Ouzou. Algeria \\ *Corresponding author: sadani.idir@yahoo.fr
}

Received June 24, 2015; Revised August 25, 2015; Accepted September 19, 2015

\begin{abstract}
We study the properties of prime number sequences obtained using a well-defined equivalence relation $\mathcal{R}$. It will be seen that the elements of each class $\dot{p}$ of $\mathcal{R}$ are all prime numbers which constitute the fundamental object of our study. The number of prime numbers of each class less than or equal to a given quantity $x$, the number of the different equivalence classes and some other results will be deduced.
\end{abstract}

Keywords: distribution of prime numbers, equivalence relation, iteration, asymptotic formula

Cite This Article: Idir Sadani, "Study of Some Sequences of Prime Numbers Defined by Iteration." Turkish Journal of Analysis and Number Theory, vol. 3, no. 4 (2015): 97-103. doi: 10.12691/tjant-3-4-2.

\section{Introduction}

Let $\mathbb{P}$ be the set of prime numbers, and for all $\mathbb{R}$, let $\pi(x)$ denote the number of prime numbers less than or equal to $x$. The prime number theorem which was shown independently by de la Vallée Poussin [1], and Hadamard [2] in 1896, states that:

$$
\pi(x) \sim \frac{x}{\ln x}, \text { as } x \rightarrow+\infty
$$

or

$$
\pi(x) \approx \operatorname{Li}(x), \text { as } x \rightarrow+\infty,
$$

where is the logarithmic integral of $x$ defined by:

$$
\operatorname{Li}(x)=\lim _{\varepsilon \rightarrow 0}\left[\int_{0}^{1-\varepsilon} \frac{d y}{\ln y}+\int_{1+\varepsilon}^{x} \frac{d y}{\ln y}\right] .
$$

We can give an equivalent statement for this theorem as, for example, let denote the n'th prime number. Then

$$
\pi^{-1}(n)=p_{n}=L i^{-1}(n) \sim n \ln n \text { as } n \rightarrow+\infty .
$$

One of our objectives here is to use a restriction of the function $\pi$ to $\mathbb{P}$ to study intrinsic properties of some sequences of primes defined by iterations. The point of departure for this study is the construction of an equivalence relation that we denote by $\mathcal{R}$. The purpose of this equivalence relation is to show first, that there is a recurrence relation between prime numbers which can be arranged in an infinity of well-defined classes dependent on the initial value. Second, the use of its properties with the famous prime number theorem is one way to find and prove other results for the possible applications in number theory. Part of our motivation came from the prime number theorem and the Riemann hypothesis. Also, it was an attempt to establish the relationship between these prime numbers classes and several as-yet unproved conjectures, such as Goldbach's Conjecture and Twin Prime Conjecture.

The structure of this paper is as follows. In section 2, we begin with the definition of our equivalence relation and its equivalence classes $\dot{p}$ where $p$ is a prime number, which constitute the fundamental object of study, and we propose some preliminary results. In section 3 , we exhibit the main results of this work by introducing and studying the functions $\pi_{i t e r}, \eta, \pi_{c}, \vartheta$ and $\theta_{0}$. The methods used here are part of elementary number theory and we have attempted to present the ideas in as elementary a way as possible. Finally, in section 4, we give some open questions related this subject.

\section{Notation}

1) We set

$$
\pi(x)=\sum_{p \leq x} 1,
$$

this function count the number of primes less than $x$.

2) We define the following functions:

$$
\begin{gathered}
\pi^{n}(x)=\overbrace{\pi \circ \pi \circ \ldots \circ \pi(x)}^{n \text { times }}, \\
\pi^{-n}(y)=\overbrace{\pi^{-1} \circ \pi^{-1} \circ \ldots \circ \pi^{-1}(y),}^{n \text { times }}
\end{gathered}
$$

where $o$ is the composition operator.

3) In many situations, we search to estimate $\Sigma$ ), where $\mathbb{P}$. Then, we use the following formula:

$$
\sum_{p \leq x} f(p) \approx \sum_{n \leq x} \frac{f(n)}{\ln n} \approx \int_{2}^{x} \frac{f(t)}{\ln t} d t .
$$

\section{Preliminary results}

\subsection{The Classes $\dot{\boldsymbol{p}}$ and Its Elements}

We start with the following lemma: 
Lemma 2.1. Let $\left.\pi\right|_{\mathbb{P}}$ be the restriction of $\pi$ to $\mathbb{P}$. Then, $\left.\pi\right|_{\mathbb{P}}: \mathbb{P} \rightarrow \mathbb{N}$ is a bijection and its inverse function is $\left.\pi\right|_{\mathbb{P}} ^{-1}: \mathbb{N} \rightarrow \mathbb{P}$.

Notation. Throughout this paper, we simply use the notation $\pi$ to designate the restriction of $\pi$ to the set $\mathbb{P}$ instead of using $\left.\pi\right|_{\mathbb{P}}$.

The proof of the following theorem is obvious.

Theorem 2.1. We define the relation $\mathcal{R}$ on the set of prime numbers $\mathbb{P}$ defined by: if $p$ and $q$ are two prime numbers, $p \mathcal{R} q$ if and only if:

1) $\pi^{0}(p)=p$ for any prime number $p$,

2) there exists $n \in \mathbb{Z}$ such that $p=\pi^{n}(q)$.

Then, $\mathcal{R}$ is an equivalence relation.

Notation. The elements of the equivalence class $\dot{p}$ are defined by:

$$
\dot{p}=\left\{\ldots, \pi^{2}(p), \pi(p), p, \pi^{-1}(p), \pi^{-2}(p), \ldots\right\} .
$$

The smallest element of $\dot{p}$, which we denote by $p_{0}$, is called the origin of the class $\dot{p}$. Then, in this case, we note that the sets $\dot{p}$ and $\dot{p}_{0}$ have the same elements.

\section{Example 1.}

$$
\dot{2}=\{2,3,5,11,31,127, \ldots\}, \dot{7}=\{7,17,59, \ldots\} .
$$

Notation. We denote by $\mathcal{P}_{0}$ the set of all origins $p_{0}$. The set $\mathcal{P}_{0}$ is given explicitly by:

$$
\mathcal{P}_{0}=\{2,7,13,19,23,29,37,43 \ldots\},
$$

and we denote by $\mathcal{P}_{0, x}$ the set of all origins less than or equal to $x$.

Theorem 2.2. Let $p$ be a prime number. Then, $\pi(p)$ is not a prime number implies that, for all $q<p$, there is no an integer $n$ such that $p=\pi^{-n}(q)$.

Proof. Let $p \in \mathbb{P}$. By the prime number theorem, $\pi^{-1}(p)$ represents the $p$-th prime number which we denote by $\mathrm{p}_{p}=\pi^{-1}(p)$.

Next, $\mathrm{p}_{p}=\pi^{-1}(p)$, implies that $\pi^{-1}\left(\mathrm{p}_{p}\right)=\pi^{-1}\left(\pi^{-1}(p)\right)$, which is the $\pi^{-1}(p)$-th prime number, so we obtain the formula:

$$
\pi^{-1}\left(\mathrm{p}_{p}\right)=\pi^{-1}\left(\pi^{-1}(p)\right)=\pi^{-2}(p)=\mathrm{p}_{\pi^{-1}(p)} .
$$

Now, we use the equation (4) which composed by $\pi^{-1}$ gives

$$
\mathrm{p}_{\pi^{-1}(p)}=\pi^{-2}(p) \Rightarrow \pi^{-1}\left(\mathrm{p}_{\pi^{-1}(p)}\right)=\pi^{-1}\left(\pi^{-2}(p)\right),
$$

that is the $\pi^{-2}(p)$-th prime number. We can write

$$
\pi^{-1}\left(\mathrm{p}_{\pi^{-1}(p)}\right)=\pi^{-1}\left(\pi^{-2}(p)\right)=\mathrm{p}_{\pi^{-2}(p)}=\pi^{-3}(p)
$$

Inductively, we obtain the following general formula:

$$
\mathrm{p}_{\pi^{-(n-1)}(p)}=\pi^{-n}(p), n \geq 1 .
$$

To each number $p$, we associate the set $A_{p}$ :

$$
A_{p}=\left\{\pi^{-n}(p), n \geq 0\right\}=\left\{p, \pi^{-1}(p), \pi^{-2}(p), \ldots\right\} .
$$

Now, suppose that $\pi(p)$ is not a prime number, we must prove that for all $q<p$, there is no $n \in \mathbb{N}$ such that $p=\pi^{-n}(q)$. To show this, suppose that there exists a positive integer $k$ such that $\pi^{-k}(q) \in A_{p}$, i.e., $\pi^{-k}(q)=$ $\pi^{-i}(p), k \geq i+1, i \in \mathbb{N}$ and we show that $\pi(p)$ is a prime number. Hence, we have, $\pi^{-k}(q)=\pi^{-i}(p)$ if and only if $p=\pi^{i-k}(q)$, which implies that $\pi(p)=\pi\left(\pi^{i-k}(q)\right)=$ $\pi^{(i+1)-k}(q), k \geq i+1$.

But, for all $k \geq i+1, \pi^{(i+1)-k}(q)=\pi(p)$ is prime, which proves what we wanted.

Remark. The cardinality of the set $A_{p}$ is infinite.

Theorem 2.3. There exists a partition $P$ of the set of prime numbers $\mathbb{P}$ defined by

$$
P=\left\{A_{p_{1}}, A_{p_{2}}, \ldots, A_{p_{n}}, \ldots\right\},
$$

such that $A_{p_{i}}=\left\{p_{i}, \pi^{-1}\left(p_{i}\right), \pi^{-2}\left(p_{i}\right), \ldots\right\}$, where $p_{i} \in \mathbb{P}$ and $\pi\left(p_{i}\right)$ is not prime number.

Proof. On the one hand, according to the prime number theorem, the number of prime numbers belonging to $[\pi(p), p]$ is equal to $\pi(p)-\pi^{2}(p)$. On the other hand, by the Theorem 2.2, there are $\pi(p)-\pi^{2}(p)-1$ prime numbers which do not belong to $A_{p}=\left\{p, \pi^{-1}(p), \pi^{-2}(p), \ldots\right\}$. We denote these numbers by $p_{1}, p_{2}, \ldots, p_{j}$, where $j=\pi(p)-\pi^{2}(p)-1$. So, we obtain $\pi(p)-\pi^{2}(p)-1$ of sets $A_{p_{k}}$ which are defined by:

$$
A_{p_{k}}=\left\{p_{k}, \pi^{-1}\left(p_{k}\right), \ldots, \pi^{-l} p_{k}\left(p_{k}\right)\right\},
$$

such that $\sum_{k \leq j} l_{p_{k}}=\pi(p)-\pi^{2}(p)-1$ and $\pi^{-l_{p_{k}}}\left(p_{k}\right)<$ $p, \forall k \leq j$. Finally, it is not difficult to see that the sets $A_{p_{k}}, k \leq j$ constitute a partition of the set of prime numbers less than or equal to $p$. So, since $p$ is arbitrary in $\mathbb{P}$, letting $p$ tend to $+\infty$ we obtain an infinity of sets $A_{p_{k}}$ which then form a partition $P$ of the set $\mathbb{P}$.

Theorem 2.4. Let $A$ be a finite set of prime numbers defined by

$$
A=\left\{p_{1}, p_{2}, \ldots, p_{n}\right\},
$$

such that $5 \leq p_{i}<p_{i+1}, 1 \leq i \leq n$ are consecutive. Then, there exists at least one set $\dot{p} \subset A$ where $p \in A$, such that $\dot{p}=\{p\}$.

Before giving the proof of this result, we give an illustrative example.

Example 2. Let $A$ be a set which defined by

$$
A=\{5,7,11,13,17\} \text {. }
$$

- The class of the integer 5 is $\dot{5}=\{5,11\}$ and its cardinality is greater than 1 .

- We notice that $7 \notin \dot{5}$, therefore the integer 7 constitutes the origin of a new class which is $\dot{7}=\{7,17\}$ and its cardinality is greater than 1 . It only remains to see that the prime number 13 does not belong to the two classes $\dot{2}$ and $\dot{7}$. Thus the prime number 13 constitutes the origin of a new class 13 and clearly its cardinality is 1 .

Proof of theorem 2.4. We suppose that each class $\dot{p}_{i}, p_{1} \leq p_{i} \leq p_{n}$ containing at least two elements i.e., the cardinality of $\dot{p}_{i}$ is equal or greater than 2 , and we suppose that $\pi\left(p_{n}\right)$ is prime. According to the theorem of prime numbers, the interval $\left[\pi\left(p_{n}\right), p_{n}\right]$ contains $\pi\left(p_{n}\right)-$ $\pi^{2}\left(p_{n}\right)=k$ prime numbers. This leads to the two following cases:

- If $k=1$, i.e., there exists only one prime number in $\left[\pi\left(p_{n}\right), p_{n}\right]$, namely $q$. Therefore, we obtain $\pi^{-1}(q) \notin A$ and $\pi(q)$ is not a prime number since $\pi(q)$ and $\pi\left(p_{n}\right)$ are 
consecutive. Then $\dot{q}$ contains only one element in $A$ which is the prime number $q$.

- If $k>1$, i.e., there exist at least two prime numbers in $\left[\pi\left(p_{n}\right), p_{n}\right]$, namely $q_{1}, \ldots, q_{k}$. We suppose that $q_{t}$ where $t \in\{1, \ldots, k\}$, is a prime number. Then $q_{t+1}$ is not prime and since $\pi^{-1}\left(q_{t+1}\right) \notin A$, then, the unique element of $\dot{q}_{t+1}$ is $q_{t+1}$.

Finally, in both cases, there exist at least one class $\dot{p}$ where $p \in A$, such that $p$ is a unique element of this class in $A$, i.e., $\dot{p}=\{p\}$. The proof now is completed.

We have the following definition:

Definition 1. Let $A$ be the set defined as in the Theorem 2.4 and $p$ is a prime number belonging to $A$. We say that $\dot{p}$ is an outside class of $A$, if

$$
\pi(p) \text { is not prime in } A \text { and } \pi^{-1}(p) \notin A \text { i.e., }|p|=1 \text { in } A \text {. }
$$

if $|\dot{p}| \geq 2$ in $A$, we say that this class is an inside class of A.

Remark. According to the Definition 1, the number $p$ is the smallest element of the class $\dot{p}$ in A, therefore, it is the origin of this class i.e., $p=p_{0}$.

\subsection{Study of $x_{n+1}=p\left(x_{n}\right)=x_{n} / \ln x_{n}$}

The function $p(x)=\frac{x}{\ln x}, x \in[e,+\infty[$, is a contraction. Therefore, the sequence $x_{n}$ defined by:

$$
x_{n+1}=p\left(x_{n}\right)=\frac{x_{n}}{\ln x_{n}},
$$

admits a fixed point. Since $x_{n}$ decreasing and is bounded below by $e \approx 2.7182818$, then it converges to the single fixed point $e$.

Notation. $p^{k}(x)=\overbrace{p \circ p \circ \ldots \circ p(x)}^{\text {k times }}$, where $\circ$ is the function composition operator.

Theorem 2.5. Let $x_{0} \geq e$ be an initial value. Then

$$
p\left(x_{0}\right)=e \prod_{i=0}^{\infty} \ln p\left(x_{i}\right)=e \prod_{i=0}^{\infty} \ln p^{i+1}\left(x_{0}\right),
$$

Proof. We set $\ln \ln x=\ln _{2} x$ and we have

$$
\begin{gathered}
x_{1}=p\left(x_{0}\right)=\frac{x_{0}}{\ln x_{0}} \Rightarrow \ln x_{1}=\ln x_{0}-\ln _{2} x_{0} \\
x_{2}=p\left(x_{1}\right)=\frac{x_{1}}{\ln x_{1}} \Rightarrow \ln x_{2}=\ln x_{1}-\ln _{2} x_{1} \\
\vdots \\
x_{n+1}=p\left(x_{n}\right)=\frac{x_{n}}{\ln x_{n}} \Rightarrow \ln x_{n+1}=\ln x_{n}-\ln _{2} x_{n} .
\end{gathered}
$$

And combining all these, we obtain

$$
\ln x_{n+1}=\ln p\left(x_{n}\right)=\ln x_{0}-\sum_{i=0}^{n} \ln _{2} x_{i} .
$$

Which is equivalent to

$$
\frac{x_{0}}{x_{n+1}}=\prod_{i=0}^{n} \ln x_{i} .
$$

Then, passing to the limit, we obtain

$$
\begin{aligned}
& \lim _{n \rightarrow+\infty} \frac{x_{0}}{x_{n+1}}=\lim _{n \rightarrow \infty} \frac{x_{0}}{p\left(x_{n}\right)}=\lim _{n \rightarrow+\infty} \prod_{i=0}^{n} \ln x_{i} \\
& =\lim _{n \rightarrow \infty} \ln x_{0} \prod_{i=0}^{n-1} \ln \ln p\left(x_{i}\right) .
\end{aligned}
$$

Consequently,

$$
\frac{x_{0}}{e}=\prod_{i=0}^{\infty} \ln x_{i}=\ln x_{0} \prod_{i=0}^{\infty} \ln p\left(x_{i}\right),
$$

and since

$$
\lim _{n \rightarrow+\infty} x_{n}=e,
$$

the formula (10) is obviously equivalent to

$$
p\left(x_{0}\right)=e \prod_{i=0}^{\infty} \ln p\left(x_{i}\right) .
$$

Which is the desired result.

Lemma 2.2. Let $x_{n+1}=p\left(x_{n}\right)$ be the sequence which is decreasing and bounded below by $e$, and let $\rho>e$ be a real number. Then, the number of iterations, which denote by $n_{i t e r}$, is depend on $\rho$ and the initial value $x_{0}=x$, and given by:

$$
n_{\text {iter }}(x, \rho) \sim-\frac{\ln c}{\ln \ln \xi_{0}}+\frac{\ln x}{\ln \ln \xi_{0}}, \text { as } x \rightarrow+\infty,
$$

where $c>0$ is a bounded value, and $\xi_{0}=\xi_{0}(x)<x$, i.e., $\xi_{0}$ depend on $x$.

Proof. We have

$$
\left|x_{2}-x_{1}\right|=\left|p^{\prime}\left(\xi_{1}\right)\right|\left|x_{1}-x_{0}\right|, \xi_{1} \in\left[x_{1}, x_{0}\right],
$$

where $p^{\prime}$ is the derivative of the function $p$.

$$
\left|x_{3}-x_{2}\right|=\left|p^{\prime}\left(\xi_{2}\right)\right|\left|x_{2}-x_{1}\right|=\left|p^{\prime}\left(\xi_{2}\right)\right|\left|p^{\prime}\left(\xi_{1}\right)\right|\left|x_{1}-x_{0}\right|,
$$

where, $\xi_{2} \in\left[x_{2}, x_{1}\right]$. Inductively, we obtain

$$
\left|x_{n}-x_{n+1}\right|=\left(\prod_{i=1}^{n} p^{\prime}\left(\xi_{i}\right)\right) \cdot\left|x_{1}-x_{0}\right|, \xi_{n} \in\left[x_{n}, x_{n-1}\right] .
$$

Next, there exists a real number $\xi_{0} \in\left[\xi_{n}, \xi_{1}\right]$, such that

$$
\left|x_{n+1}-x_{n}\right|=\left|p^{\prime}\left(\xi_{0}\right)\right|^{n} \cdot\left|x_{1}-x_{0}\right|
$$

Or in an equivalent way, since, $x_{n}>x_{n+1}, \forall n \in \mathbb{N}$ and $x>e$,

$$
x_{n}-x_{n+1}=\left(p^{\prime}\left(\xi_{0}\right)\right)^{n} \cdot\left(x_{0}-x_{1}\right) .
$$

We can extract the value of $n$ from the above equality:

$$
n=\frac{\ln \left(x_{n}-x_{n+1}\right)}{\ln p^{\prime}\left(\xi_{0}\right)}-\frac{\ln \left(x_{0}-x_{1}\right)}{\ln p^{\prime}\left(\xi_{0}\right)},
$$

and replacing $p^{\prime}$ by its value, we get

$$
n_{\text {iter }}=\frac{\ln \left(x_{n}-x_{n+1}\right)}{\ln \left(\ln \xi_{0}-1\right)-2 \ln \ln \xi_{0}}-\frac{\ln \left(x_{0}-x_{1}\right)}{\ln \left(\ln \xi_{0}-1\right)-2 \ln \ln \xi_{0}}
$$

Now, according to the definition of $\rho$, the sequences $x_{n}$ and $x_{n+1}$ are bounded, then it holds for the difference 
$x_{n}-x_{n+1}$ which we denote it by $c$. Then, we have, by setting $x_{0}=x$ and $n=n_{\text {iter }}(x, \rho)$ :

$$
\begin{aligned}
n_{\text {iter }}(x, \rho)= & \frac{\ln c}{\ln \left(\ln \xi_{0}-1\right)-2 \ln \ln \xi_{0}} \\
& -\frac{\ln \left(x-x_{1}\right)}{\ln \left(\ln \xi_{0}-1\right)-2 \ln \ln \xi_{0}}
\end{aligned}
$$

Finally, we have the following limits:

$$
\xi_{0} \rightarrow+\infty \text { as } x \rightarrow+\infty \text {, }
$$

$$
\ln \left(\ln \xi_{0}-1\right)-2 \ln \ln \xi_{0} \rightarrow-\ln \ln \xi_{0} \text { as } x \rightarrow+\infty,
$$

$$
x-x_{1} \rightarrow x \text { as } x \rightarrow+\infty,
$$

then,

$$
n_{i t e r}(x, \rho) \sim-\frac{\ln c}{\ln \ln \xi_{0}}+\frac{\ln x}{\ln \ln \xi_{0}}, \text { as } x \rightarrow+\infty .
$$

\section{Principal Results}

\subsection{Definition and Estimate of the Functions $\boldsymbol{\pi}_{\text {iter }}, \boldsymbol{\eta}$ and $\boldsymbol{\vartheta}$}

We set the following definition:

Definition 2. Let $x \in \mathbb{R}, x>0$. We define

$$
\begin{gathered}
\pi_{\text {iter }}\left(x, p_{0}\right)=\sum_{\substack{p_{0} \leq p \leq x \\
p \in \dot{p}_{0}}} 1=\sum_{\substack{p_{0} \leq p \leq x \\
p=\pi^{-l}\left(p_{0}\right)}} 1, l \in \mathbb{N} .
\end{gathered}
$$

Then $\pi_{i t e r}\left(x, p_{0}\right)$ counts the number of prime numbers less than or equal to $x$ belonging to the class $\dot{p}_{0}$.

Lemma 3.1. We have

$$
\pi_{i t e r}\left(x, p_{0}\right) \sim n_{i t e r}(x, \rho) \sim n_{i t e r}\left(x, p_{0}\right) \text {, as } x \rightarrow+\infty .
$$

Proof. According to the definition of $\rho$, there exists $n \in \mathbb{N}$ such that $x_{n}=p^{n}(x)=\rho$. Next, from the prime number theorem, we have

$$
\pi(x) \sim p(x)=\frac{x}{\ln (x)}, x \rightarrow \infty \Rightarrow \pi^{n}(x) \sim p^{n}(x), x \rightarrow \infty .
$$

Moreover, supposing that $\pi^{n}(x)=p_{0}$ with $\pi\left(p_{0}\right)$ not a prime number, it follows that

$$
\left(p_{0}=\pi^{n}(x)\right) \sim\left(p^{n}(x)=\rho\right), x \rightarrow \infty .
$$

And,

$$
\begin{aligned}
\pi_{\text {iter }}\left(x, p_{0}\right) & =\sum_{\substack{p_{0} \leq p \leq x \\
p \in \dot{p}_{0}}} 1=\sum_{\substack{p_{0} \leq p \leq x \\
p=\pi^{-l}\left(p_{0}\right)}} 1 \sim \sum_{\substack{\rho \leq p \leq x \\
p \in \dot{p}_{0}}} 1 \sim \sum_{\substack{\rho \leq p \leq x \\
p \sim p^{-l}(\rho)}} 1 \\
& =\sum_{l=1}^{n(x, \rho)} 1=n_{\text {iter }}(x, \rho) \sim n_{\text {iter }}\left(x, p_{0}\right),
\end{aligned}
$$

as $x \rightarrow \infty$.

Definition 3. Let $x>0$. We define the functions $\eta\left(x, p_{0}\right)$ and $\vartheta\left(x, p_{0}\right)$ as follows:

$$
\eta\left(x, p_{0}\right)=\sum_{\substack{p_{0} \leq p \leq x \\ p \in \dot{p}_{0}}} \ln \ln p .
$$

$$
\vartheta\left(x, p_{0}\right)=\sum_{p \leq x, p \in \dot{p}_{0}} \frac{\ln \ln x}{\ln \ln p} \ln \ln p .
$$

Theorem 3.1. We have

$$
\eta\left(x, p_{0}\right) \sim \ln x+o(\ln x) .
$$

Proof. In view of the proof of Lemma 3.1, we have,

$$
\ln x_{0} \prod_{i=0}^{n} \ln \pi\left(x_{i}\right) \sim \ln x_{0} \prod_{i=0}^{n} \ln p\left(x_{i}\right) \sim \frac{x_{0}}{p_{0}}=\frac{x_{0}}{p_{0}},
$$

which is equivalent to

$$
\begin{aligned}
& \ln \ln x_{0}+\sum_{i=0}^{n} \ln \ln \pi\left(x_{i}\right)=\ln \ln x+\sum_{i=0}^{n} \ln \ln \pi\left(x_{i}\right) \\
& =\sum_{\substack{p_{0} \leq p \leq x \\
p \in \dot{p}_{0}}} \ln \ln p \sim \ln x-\ln p_{0} .
\end{aligned}
$$

Finally, for all fixed $p_{0}$ and $x$ tend to infinity, we have $-\ln p_{0} / \ln x \rightarrow 0$, then

$$
\sum_{\substack{p_{0} \leq p \leq x \\ p \in \dot{p}_{0}}} \ln \ln p \sim \ln x+o(\ln x) .
$$

i.e., $\eta\left(x, p_{0}\right) \sim \ln x+o(\ln x)$.

Theorem 3.2. We have,

$$
\begin{aligned}
& \text { 1) } \pi_{\text {iter }}\left(x, p_{0}\right) \sim \frac{\eta\left(x, p_{0}\right)}{\ln \ln x}, x \rightarrow \infty \\
& \text { 2) } \pi_{\text {iter }}\left(x, p_{0}\right) \sim \pi_{\text {iter }}(x) \sim \frac{\ln x}{\ln \ln x}+o\left(\frac{\ln x}{\ln \ln x}\right), x \rightarrow \infty \\
& \text { 3) } \pi_{\text {iter }}\left(x, p_{0}\right)=\frac{\vartheta\left(x, p_{0}\right)}{\ln \ln x}+O\left(\frac{\ln x}{\ln \ln x}\right), x \rightarrow \infty .
\end{aligned}
$$

Proof.

1) For the proof of the first formula, we have, on the one hand

$$
\begin{aligned}
\eta\left(x, p_{0}\right) & =\sum_{\substack{p_{0} \leq p \leq x \\
p \in \dot{p}_{0}}} \ln \ln p \leq \ln \ln x \sum_{\substack{p_{0} \leq p \leq x \\
p \in \dot{p}_{0}}} 1 \\
& =\pi_{\text {iter }}\left(x, p_{0}\right) \ln \ln x .
\end{aligned}
$$

Then we obtain

$$
\pi_{i t e r}\left(x, p_{0}\right)>\frac{\eta\left(x, p_{0}\right)}{\ln \ln x} .
$$

On the other hand, for all $x>e, x^{\delta}>p_{0}$ with $0<\delta<$ 1 , we have

$$
\begin{aligned}
& \eta\left(x, p_{0}\right) \geq \ln x^{\delta} \sum_{x^{\delta} \leq p \leq x} 1=(\ln \delta+\mathrm{n} \ln x) \sum_{\substack{p_{0} \leq p \leq x \\
p \in \dot{p}_{0}}} 1 \\
& p \in \dot{p}_{0} \quad p \in \dot{p}_{0} \\
& =(\ln \delta+\ln \ln x)\left(\pi_{i t e r}\left(x, p_{0}\right)-\pi_{i t e r}\left(x^{\delta}, p_{0}\right)\right) \\
& \geq(\ln x)^{\delta}+\frac{\eta\left(x, p_{0}\right)}{\ln \delta+\ln \ln x}
\end{aligned}
$$

Then 


$$
\pi_{i t e r}\left(x, p_{0}\right) \leq(\ln x)^{\delta}+\frac{\eta\left(x, p_{0}\right)}{\ln \delta+\ln \ln x} .
$$

Now, according to Lemma

3.1 , $(\ln x)^{\delta}=o\left(\pi_{i t e r}\left(x, p_{0}\right)\right)$, and then for $x$ sufficiently large (depending on $\delta),(\ln x)^{\delta} \leq(1-\delta) \pi_{i t e r}\left(x, p_{0}\right)$, and thus

$$
\pi_{i t e r}\left(x, p_{0}\right) \leq \frac{\eta\left(x, p_{0}\right)}{\delta(\ln \delta+\ln \ln x)} .
$$

Now, for all $\varepsilon>0$, we can choose $\delta$ more near to 1 . For this $\delta$, so that $\frac{1}{\delta}=\varepsilon+1$, and for x sufficiently large, we have

$$
\pi_{i t e r}\left(x, p_{0}\right)<(1+\varepsilon) \frac{\eta\left(x, p_{0}\right)}{\ln \ln x} .
$$

2) According to Theorem 3.1, we have

$$
\begin{aligned}
& \pi_{\text {iter }}\left(x, p_{0}\right) \sim \frac{\eta\left(x, p_{0}\right)}{\ln \ln x} \sim \frac{\ln x}{\ln \ln x}+o\left(\frac{\ln x}{\ln \ln x}\right) \\
& \sim \pi_{\text {iter }}(x), x \rightarrow \infty .
\end{aligned}
$$

3) Concerning the third equality, we have

$$
\vartheta\left(x, p_{0}\right) \leq \pi_{\text {iter }}\left(x, p_{0}\right) \ln \ln x .
$$

Evaluate now the difference

$$
\pi_{\text {iter }}\left(x, p_{0}\right) \ln \ln x-\sum_{p \leq x, p \in \dot{p}_{0}}\left\lfloor\frac{\ln \ln x}{\ln \ln p}\right\rfloor \ln \ln p .
$$

Then

$$
\begin{aligned}
& \pi_{\text {iter }}\left(x, p_{0}\right) \ln \ln x-\sum_{p \leq x, p \in \dot{p}_{0}}\left\lfloor\frac{\ln \ln x}{\ln \ln p}\right\rfloor \ln \ln p \\
= & \sum_{p \leq x, p \in \dot{p}_{0}}\left(\ln \ln x-\left\lfloor\frac{\ln \ln x}{\ln \ln p}\right\rfloor \ln \ln p\right) \\
= & \sum_{p \leq \sqrt{x}, p \in \dot{p}_{0}}\left(\ln \ln x-\left\lfloor\frac{\ln \ln x}{\ln \ln p}\right\rfloor \ln \ln p\right) \\
+ & \sum_{\sqrt{x} \leq p \leq x, p \in \dot{p}_{0}}\left(\ln \ln x-\left\lfloor\frac{\ln \ln x}{\ln \ln p}\right\rfloor \ln \ln p\right) \\
\leq & \sum_{p \leq \sqrt{x}, p \in \dot{p}_{0}}\left\{\frac{\ln \ln x}{\ln \ln p}\right\} \ln \ln p \\
+ & \sum_{\sqrt{x} \leq p \leq x, p \in \dot{p}_{0}}(\ln \ln x-\ln \ln p) \\
\leq & \vartheta\left(\sqrt{x}, p_{0}\right)+\sum_{\sqrt{x} \leq p \leq x, p \in \dot{p}_{0}} \int_{p}^{x} \frac{d t}{t \ln t} \\
\leq & \vartheta\left(\sqrt{x}, p_{0}\right)+\int_{\sqrt{x}}^{x} \sum_{\sqrt{x} \leq p \leq x, p \in \dot{p}_{0}} 1_{\{p \leq t\}} \frac{d t}{t \ln t}
\end{aligned}
$$

However, for $t \in[\sqrt{x}, x]$ :

$$
\sum_{\sqrt{x} \leq p \leq x, p \in \dot{p}_{0}} 1_{\{p \leq t\}}=\sum_{p \leq x, p \in \dot{p}_{0}} 1_{\{p \leq t\}}-\sum_{p \leq \sqrt{x}, p \in \dot{p}_{0}} 1_{\{p \leq t\}}
$$

Then,

$$
\sum_{p \leq t} 1-\sum_{p \leq \sqrt{x}} 1=\pi_{i t e r}\left(t, p_{0}\right)-\pi_{i t e r}\left(\sqrt{x}, p_{0}\right) \leq \pi_{i t e r}\left(t, p_{0}\right)
$$

thus

$$
\begin{aligned}
& \pi_{\text {iter }}\left(x, p_{0}\right) \ln \ln x-\vartheta(x) \\
& \leq \vartheta\left(\sqrt{x}, p_{0}\right)+\int_{\sqrt{x}}^{x} \frac{\pi_{i t e r}\left(t, p_{0}\right)}{t \ln \ln t} d t \\
& \leq \vartheta\left(\sqrt{x}, p_{0}\right)+\int_{\sqrt{x}}^{x} \frac{2 \ln t}{t \ln t \ln \ln t} d t \\
& \leq \vartheta\left(\sqrt{x}, p_{0}\right)+\int_{\sqrt{x}}^{x} \frac{2}{t \ln \ln t} d t .
\end{aligned}
$$

By using $\vartheta\left(x, p_{0}\right) \leq 2 \ln x$, since

$$
\vartheta\left(x, p_{0}\right) \leq \pi_{\text {iter }}\left(x, p_{0}\right) \ln \ln x \leq \frac{\ln x}{\ln \ln x} \ln \ln x=\ln x .
$$

we obtain

$$
\begin{gathered}
\pi_{\text {iter }}\left(x, p_{0}\right) \ln \ln x-\vartheta\left(x, p_{0}\right) \leq O(\ln \sqrt{x})+O\left(\frac{\ln x}{\ln \ln x}\right) \\
\text { Then, } \pi_{\text {iter }}\left(x, p_{0}\right)=\frac{\vartheta\left(x, p_{0}\right)}{\ln \ln x}+O\left(\frac{\ln x}{\ln \ln x}\right) .
\end{gathered}
$$

\subsection{Definition and Estimate of $\pi_{c}$ and $\theta_{0}$}

\subsubsection{Definition and Estimate of $\pi_{c}$}

\section{Definition 4.}

1. Let $x$ be a positive real number. We denote by $\pi_{c}(x)$ the number of different classes $\dot{p}$ such that $2 \leq p \leq x$. Precisely,

$$
\pi_{c}(x):=\sum_{p_{0} \leq x} 1, p_{0} \in \mathcal{P}_{0} .
$$

2. We denote by $\theta_{0}(x)$ the function defined by

$$
\theta_{0}(x)=\sum_{p_{0} \leq x} \ln p_{0} .
$$

Example 3. In $[2,11]$ ?, the value 2 represents the origin of the class $\dot{2}$ but the values 3,5,11 do not, since they belong to the same class $\dot{2}$. The value 7 represent the origin of the class $\dot{7}$. Thus in this case, we have $\pi_{c}(x)=2$.

We have the following result:

Theorem 3.3. We have,

$$
\lim _{x \rightarrow \infty} \pi_{c}(x)=+\infty
$$

Proof. Suppose that the number of different classes is finite as $x \rightarrow \infty$. We know that

$$
\sum_{i=0}^{\infty} \frac{1}{p_{i}}=\infty
$$

Next, let $p_{i}^{k} \in \dot{p}_{k}$, where $k$ is finite by hypothesis. We obtain

$$
\sum_{i=0}^{\infty} \frac{1}{p_{i}}=\sum_{k<\infty} \sum_{i=0}^{\infty} \frac{1}{p_{i}^{k}}
$$

Therefore, we have $\sum_{i=0}^{\infty} \frac{1}{p_{i}^{k}}<\infty$, and since the second sum has a finite number of terms, we deduce 


$$
\sum_{k<\infty} \sum_{i=0}^{\infty} \frac{1}{p_{i}^{k}}<\infty
$$

which contradicts formula (13).

Theorem 3.4. Let $p_{0} \in[2, x]$. Then

$$
\pi_{c}(x)=\pi(x)-\pi(\pi(x)) \text {. }
$$

Proof. To find the value of $\pi_{c}(x)$ means that we estimate the number of origins $p_{0}$. Clearly, the prime number $p_{0}$ is an origin that means $\pi\left(p_{0}\right)$ is not a prime number. Then, let $p_{0}$ be between 2 and $x$, and let $p_{0, x} \in \mathcal{P}_{0}$ be the greatest prime number in $[2, x]$, therefore $\pi\left(p_{0, x}\right)$ is not a prime number and for all integer $l \geq 1, \pi^{-l}\left(\pi\left(p_{0, x}\right)+\right.$ 1) $>p_{0, x}$. So, we only have to search the numbers which are not primes and less than $\pi\left(p_{0, x}\right)$. Thus, we have

$1^{\circ}$ The number of the even numbers less than or equal to $p_{0, x}$ equal to $\frac{\pi\left(p_{0, x}\right)}{2}$.

$2^{\circ}$ The number of the odd numbers less than or equal to $p_{0, x}$ equal to $\frac{\pi\left(p_{0, x}\right)}{2}-\pi\left(\pi\left(p_{0, x}\right)\right)$ such that $\pi\left(\pi\left(p_{0, x}\right)\right)$ is the number of the prime numbers less than or equal to $p_{0, x}$.

Next, we add the two quantities, we obtain, since $p_{0, x} \leq x$, the quantity $\pi_{c}(x)$ which is equal to

$$
\pi_{c}(x)=\pi(x)-\pi(\pi(x)) .
$$

\subsubsection{Estimate of $\boldsymbol{\theta}_{0}$}

For all initial value $y_{0}>e$, we define the following sequence:

$$
y_{n+1}=y_{n} \ln y_{n} .
$$

It is not difficult to see that, this sequence is stationary for $y_{0}=e$ and increasing divergent to infinite for $y_{0}>e$ and as $n \rightarrow \infty$. It is clear that, inductively, $y_{n} \geq y_{0}\left(\ln y_{0} \times\right.$ $\left.\ln y_{0} \times \ldots \times \ln y_{0}\right)=y_{0}\left(\ln y_{0}\right)^{n}$, then we have the following consequence:

Lemma 3.2. We have,

$$
\pi_{c}(x) \leq \frac{x \ln \ln x}{\ln ^{2} x}+\frac{\sum_{p_{0} \leq x} \ln p_{0}}{\ln x}=\frac{x \ln \ln x}{\ln ^{2} x}+\frac{\theta_{0}(x)}{\ln x},
$$

where $p_{0}$ represents the origin of the classes $\dot{p}$.

Proof.. Since for all $p=p_{n}, n>0$, we have $p>p_{0} \ln ^{n} p_{0}$, then

$$
\begin{aligned}
& \sum_{p \leq x} \ln \ln p \geq \sum_{p_{0} \ln ^{n} p_{0} \leq x, n} \ln \ln p_{0}=\sum_{p_{0}, n \leq \frac{\ln x-\ln p_{0}}{\ln \ln p_{0}}} \ln \ln p_{0} \\
& =\sum_{p_{0} \leq x} \ln \ln p_{0} \frac{\ln x-\ln p_{0}}{\ln \ln p_{0}} \sim \sum_{p_{0} \leq x}\left(\ln x-\ln p_{0}\right) \\
& =(\ln x) \pi_{\text {iter }}(x)-\sum_{p_{0} \leq x} \ln p_{0} .
\end{aligned}
$$

In the expression:

$$
\sum_{p_{0} \ln ^{n} p_{0} \leq x, n} \ln \ln p_{0},
$$

the value of $n$ obviously depends on $p_{0}$ and if we denote by $n_{p_{0}}$, then, we have

$$
\sum_{p_{0}} n_{p_{0}} \sim \pi(x)
$$

i.e., the number of elements of the form $p_{0} \ln ^{n} p_{0} \leq x$ must be equal to number of prime numbers $p \leq x$.

According to the formula (3), we have

$$
\sum_{p \leq x} \ln \ln p \approx \frac{x \ln \ln x}{\ln x},
$$

therefore, the inequality is obtained directly by substitution.

Proposition 3.1. We have,

$$
\begin{gathered}
x\left(1-\frac{1}{\ln x-\ln \ln x}-\frac{\ln \ln x}{\ln x}\right) \\
\leq \theta_{0}(x) \leq x\left(1-\frac{1}{\ln x-\ln \ln x}\right) \\
\theta_{0}(x) \sim x \text { as } x \rightarrow \infty .
\end{gathered}
$$

Proof.

1. We have

$$
\begin{aligned}
& \pi(x)-\pi(\pi(x)) \leq \frac{x \ln \ln x}{\ln ^{2} x}+\frac{\sum_{p_{0} \leq x} \ln p_{0}}{\ln x} \\
& \Rightarrow \sum_{p_{0} \leq x} \ln p_{0} \geq(\pi(x)-\pi(\pi(x))) \ln x-\frac{x \ln \ln x}{\ln x} .
\end{aligned}
$$

Moreover, since

$$
\sum_{p_{0} \leq x} \ln p_{0} \leq \ln x \sum_{p_{0} \leq x} 1=\pi_{c}(x) \ln x .
$$

And recalling that

$$
\pi(x) \sim \frac{x}{\ln x} .
$$

We obtain

$$
\begin{aligned}
& \sum_{p_{0} \leq x} \ln p_{0} \geq\left(\frac{x}{\ln x}-\frac{x}{\ln x(\ln x-\ln \ln x)}\right) \ln x-\frac{x \ln \ln x}{\ln x} \\
& =x\left(1-\frac{1}{\ln x-\ln \ln x}-\frac{\ln \ln x}{\ln x}\right) .
\end{aligned}
$$

The second inequality is obtained in the same way,

$$
\begin{aligned}
& \sum_{p_{0} \leq x} \ln p_{0} \leq \ln x\left(\frac{x}{\ln x}-\frac{x}{\ln x(\ln x-\ln \ln x)}\right) \\
& =x\left(1-\frac{1}{\ln x-\ln \ln x}\right) .
\end{aligned}
$$

2. It is enough to tend $x$ to $+\infty$ in the inequality (16).

\section{Conclusion and Future Work}

There is a lot of results on properties of prime numbers. There are innumerable ideas in this field regarding the randomness of prime numbers. However, it turns out that prime numbers do not appear absolutely randomly, meaning, it is not entirely true that there is no way whatsoever to see some relations and find some functions 
to generate a lenght of few prime numbers. Patterns do emerge in the distribution of primes over varied ranges of number sets. In this paper we have investigated sequences of primes obtained by the equivalence relation $\mathcal{R}$, from which we have associated various arithmetic functions. As we have seen in the sections above, many classical results stated in elementary number theory can be restated with our sequences. Also, there are many other asymptotic formulas can be deduced, we have just presented several of them as examples.

As far as motivation, the concern relates to the developing of new ideas for solving great unsolved problems and conjectures encountered in this field.

A highly intriguing area in primes is the concept of twin primes. These are prime numbers which differ by the number 2 for example: 3 and 5, 5 and 7, 11 and 13, etc. There is an attempt being made to prove that there are infinitely many twin primes that exist in the natural number system. These are the concepts that come to mind. Others are pretty much minor results.

Yitang Zhang, in his paper [3], attacked the problem by proving that the number of primes that are less than 70 million units apart is infinite. While 70 million is a long, long way away from 2 , Zhang's work marked the first time anyone was able to assign any specific proven number to the gaps between primes.

In November 2013, James Maynard, in [4] , introduced a new refinement of the GPY sieve, allowing him to reduce the bound to 600 and show that for any $m$ there exists a bounded interval containing $m$ prime numbers.

At the present time, let me explain and share some general ideas and questions about my future work. Firstly, the questions raised are very broad in scope and cannot be addressed directly. This means that, it is preferable to resort to a methodology (plan in stages) to tackle the great problems in a structured manner. Secondly, for that reason, we have proposed and developed the results of this paper (see the problem 1 and 2).

Finally, here are just a few questions and conjectures a little more direct, I think are important.

Problem 1 The twin prime conjecture is equivalent to conjecturing the translates of the 2 -tuple $(0,2)$ (the values of the pair $(n, n+2))$ are simultaneously prime values infinitely often. The question is: show that if the
2 -tuple $(n, n+6)$ are simultaneously prime values infinitely often then the $2-$ tuple $(\pi(n), \pi(n+6))$ are simultaneously twin primes infinitely often, and then the twin prime conjecture is true. We have posed this question from the perspective of finding recurrence relation between primes.

Problem 2 Let $p_{i}$ and $p_{i+2}$ be twin primes. Show that

$$
\left(\sum_{i=1}^{i=n}\left(\pi^{-1}\left(p_{i+2}\right)-\pi^{-1}\left(p_{i}\right)\right)\right)^{2} \sim O\left(n^{3} \ln ^{2} n\right) .
$$

\section{Problem 3}

1. Does the set $\mathcal{P}_{0}$ contain an infinity of twin primes?

2 . Let $p_{0} \in \mathcal{P}_{0}$ fixed. Are there an infinity of primes of the form $\pi^{-k}\left(p_{0}\right)$ such that $\pi^{-k}\left(p_{0}\right) \pm 2$ is prime?

3. Study of

$$
\sum_{p \in \mathcal{P}_{0}} \frac{1}{1-\frac{1}{p^{s}}}, \text { is a complex number }
$$

There is continuing research to prove these conjectures and questions rigorously, using the results of this paper and advanced techniques in number theory in the next work.

\section{Acknowledgement}

We sincerely thank the editor and the referees for their valuable comments and suggestions.

\section{References}

[1] C. J. de la Vallée Poussin, "Recherche analytique sur la théorie des nombres", Ann. Soc. Sci. Bruxelle, 20. 183-256. 1896.

[2] J. Hadamard, "Sur la distribution des zéros de la fonction $\zeta$ (s) et ses conséquences arithmétiques”, Bull. SOC. Math. France, 24. 199-220. 1896.

[3] Y. Zhang, “Bounded gaps between primes", Ann. of Math, 179, 1121-1174. 2014.

[4] J. Maynard, "Small gaps between primes", Ann. of Math, 181. 383-414. 2015. 